\title{
Fractal cycling
}

\section{DOI:}

10.1038/nrm2284
Cell-cycle arrest in metaphase of meiosis II in an unfertilized animal egg is maintained by high cyclindependent kinase (CDK) activity. Following fertilization, a rapid transient increase in cytosolic $\mathrm{Ca}^{2+}$ mediates exit from meiosis, which is followed by numerous events that coordinate the formation of the diploid zygote. Two separate studies, led by Tim Hunt and Keita Ohsumi, have expanded our understanding of the initial signalling events downstream of the $\mathrm{Ca}^{2+}$ transient, which has important implications for the oscillatory regulation that coordinates exit from mitosis and meiosis (M-phase). The $\mathrm{Ca}^{2+} /$ calmodulin-dependent kinase-II (CaMKII) is activated by the $\mathrm{Ca}^{2+}$ transient that follows fertilization of Xenopus laevis eggs, and CaMKII-dependent phosphorylation of Erp1 (an inhibitor of the anaphase promoting complex/cyclosome (APC/C) ubiquitin ligase) is a crucial

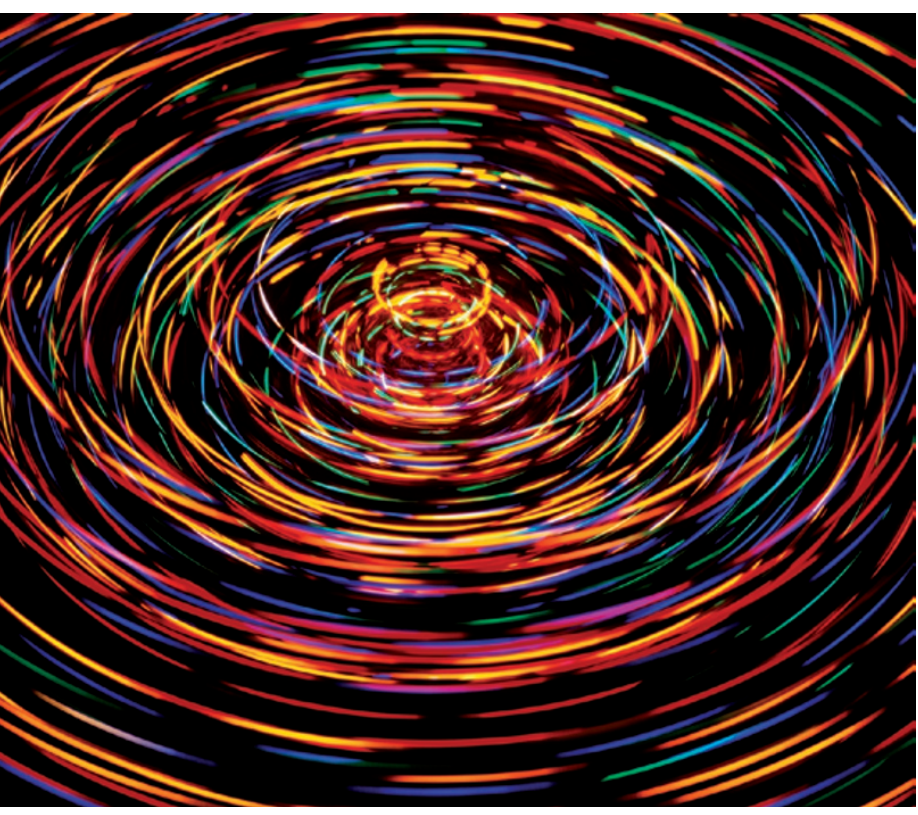

downstream event that mediates its degradation. This allows APC/C to ubiquitylate and hence target cyclins - among other proteins — for degradation, which facilitates progression through and exit from M-phase by inhibiting CDK activity.

Mochida and Hunt now show that Apc3 and Cdc20/Fizzy (components of the APC/C) are dephosphorylated following CaMKII-dependent phosphorylation of Erp1, indicating that a $\mathrm{Ca}^{2+}$-responsive phosphatase also functions in meiotic exit. Moreover, phosphatase activity increased 40-50-fold in $\mathrm{Ca}^{2+}$-treated $X$. laevis extracts, and this activity correlated with the cytosolic $\mathrm{Ca}^{2+}$ transient. The phosphatase responsible is calcineurin (also known as PP2B), inhibition of which delayed the full activation of $\mathrm{APC} / \mathrm{C}$ and hence delayed cyclin degradation in $\mathrm{Ca}^{2+}$-stimulated egg extracts.

Consistently, Ohsumi and colleagues showed that calcineurin is activated immediately after $\mathrm{Ca}^{2+}$ addition to arrested $X$. laevis egg extracts and that this occurred concomitantly with, but independently of, CaMKII activation. Inactivation of Cdk1 was delayed and could not be sustained when calcineurin was inhibited, and inhibition of both calcineurin and CaMKII prevented the formation of segregated nuclei and meiosis II exit. They suggest that calcineurin activity enhances CaMKII-mediated Erp1 degradation, facilitating full activation of the APC/C, although this remains to be determined. Nevertheless, both enzymes appear to be essential for, and synergistically inactivate, Cdk1 to exit meiosis II. They also showed that calcineurin activity negatively regulates later events that are required to form the diploid zygote, indicating that post-fertilization events are regulated, in part, by phosphatase oscillations.

So, is phosphatase activity required for the global reversion of proteins from the M-phase hyperphosphorylated state to the interphase hypophosphorylated state? Using antibodies that recognize phosphorylated CDK-consensus sequences, Mochida and Hunt showed that almost all detected phosphorylations in meiosis-II-arrested extracts were delayed by the inhibition of calcineurin. Furthermore, inhibition of calcineurin resulted in partial decondensation of chromosomes and delayed reformation of the nuclear envelope around segregated chromosomes. In addition, coexpression of constitutively active, $\mathrm{Ca}^{2+}$-independent calcineurin and CaMKII mimicked the effects of the cytosolic $\mathrm{Ca}^{2+}$ transient in fertilized $X$. laevis eggs, demonstrating that the concomitant inactivation of kinases (by APC/C) and activation of phosphatases is required for meiotic exit. Interestingly, they showed that calcineurin is not required for exit from mitosis, but oscillations of a currently unidentified phosphatase correlated with mitotic exit and entry into the proceeding interphase. These findings suggest that cycles of phosphatase activity, in combination with cycles of kinase activity and degradation, could coordinate the global regulation of cell cycling.

Gemma K. Alderton

ORIGINAL RESEARCH PAPERS Mochida, S. $\&$ Hunt, T. Calcineurin is required to release Xenopus egg extracts from meiotic M phase. Nature 449, 336-340 (2007) | Nishiyama, T. et al. Transient activation of calcineurin is essential to initiate embryonic development in Xenopus laevis. Nature 449, 341-345 (2007) 